\title{
Magnetic polarons in Eu-based films of magnetic semiconductors
}

\author{
Vyacheslav G. Storchak, ${ }^{1, *}$ Dmitry G. Eshchenko, ${ }^{2,3}$ Elvezio Morenzoni, ${ }^{3}$ Nicholas Ingle, ${ }^{4}$ Wolfgang Heiss, ${ }^{5}$ \\ Thomas Schwarzl, ${ }^{5}$ Gunther Springholz, ${ }^{5}$ R. L. Kallaher, ${ }^{6}$ and Stephan von Molnár ${ }^{6}$ \\ ${ }^{1}$ Russian Research Centre "Kurchatov Institute," Kurchatov Sq. 1, Moscow 123182, Russia \\ ${ }^{2}$ Physik-Institut der Universität Zürich, CH-8057 Zürich, Switzerland \\ ${ }^{3}$ Laboratory for Muon Spin Spectroscopy, Paul Scherrer Institute, CH-5232 Villigen, Switzerland \\ ${ }^{4}$ Department of Physics and Astronomy, University of British Columbia, Vancouver, British Columbia, Canada V6T 1Z1 \\ ${ }^{5}$ Institut für Halbleiter- und Festkörperphysik, Johannes Kepler Universität, A-404 Linz, Austria \\ ${ }^{6}$ The Center for Materials Research and Technology, Florida State University, Tallahassee, Florida 32306, USA
}

(Received 9 February 2010; published 5 April 2010; corrected 13 May 2010)

\begin{abstract}
Various films of magnetic semiconductors $\mathrm{EuO}, \mathrm{EuS}, \mathrm{EuSe}$, and EuTe have been studied by low-energy muon spin relaxation (LE- $\mu \mathrm{SR}$ ) as well as by magnetization measurement techniques. LE- $\mu \mathrm{SR}$ allows measurements of the distribution of magnetic field on the subnanometer scale unaccessible to traditional macroscopic techniques. A bound state of an electron around a positive muon-a magnetic polaron-is formed in the paramagnetic phase of Eu-based films up to room temperature. The local environment around the muon in the magnetic polaron is shown to be ferromagnetic.
\end{abstract}

DOI: 10.1103/PhysRevB.81.153201

PACS number(s): 75.50.Pp, 75.70.-i, 76.75.+i

Following a remarkable success of the metallic spintronics based on giant-magnetoresistive effect, ${ }^{1}$ considerable efforts have been invested in recent years into the less developed semiconductor spintronics with the aim to produce three-terminal spintronic devices, in particular, spin transistors. ${ }^{2,3}$ One of the most important prerequisites for such a technology is an effective injection of spin-polarized carriers from a ferromagnet (FM) into a nonmagnetic semiconductor, preferably one of those currently used in integrated circuits such as $\mathrm{Si}$ or GaAs. ${ }^{4}$ Much work on metallic FM injectors proved them to be ineffective in either ohmic regime or tunneling injection. ${ }^{5-7}$ Perhaps the best choice for the FM injector in such devices are magnetic semiconductors (MS) because of their compability with nonmagnetic semiconductors: the use of MS as a spin-polarized carrier injector avoids the so-called "conductivity mismatch" 8 which presents a fundamental difficulty for effective spin injection into a semiconductor from the FM $3 d$ transition metals.

The successful demonstration of the injection of polarized spins in spintronic devices ${ }^{9,10}$ involves Mn-doped dilute magnetic semiconductors (DMS) as spin injectors. Current progress in spintronics is mainly connected to those DMS which become ferromagnetic by addition of several percent of magnetic impurities. ${ }^{11}$ However, this addition significantly affects their homogeneity ${ }^{12-14}$ which may cause an effective spin-flip scattering of spin-polarized carriers with subsequent reduction in the effectiveness of the spin injection. This unwanted feature is absent in concentrated MS such as Eu chalcogenides and $\mathrm{EuO}$ which are intrinsically magnetic.

Being examples of strongly correlated electron systems, MS demonstrate strong dependence of the electrical and optical properties on the magnetization and spin fluctuations of the magnetic lattice ${ }^{15}$ which stimulated their extensive studies in the 1960s and 1970s. Current renaissance in the study of MS is caused by the fact that they are relatives to DMS, high-temperature superconductors and colossalmagnetoresistive manganites. ${ }^{15,16}$ Magnetic semiconductors offer several important advantages over DMS such as higher magnetization, spatial magnetic homogeneity and wider range of conductivity tunability by doping so that they can be used as spin filters in the insulating state and as spin injectors when doped. ${ }^{17-19}$

When doped, however, these materials typically enter into dominant states that are not spatially homogeneous. Early studies of MS led to development of new concepts of condensed matter physics such as magnetic polaron and magnetic phase separation. Therefore MS are of fundamental interest because they provide optimal conditions for the formation of a magnetic polaron (MP) — a few-body system comprized of conduction electron (localized on the nanoscale region of different phase which is stabilized by electron localization) and local magnetic moments of the host [e.g., as a FM "droplet" in an antiferromagnetic (AFM) or paramagnetic (PM) "sea"]. ${ }^{15,20}$ Such MP determines most of the electrical, magnetic, and optical properties of these materials. In particular, low levels of electron doping either by oxygen deficiency or the substitution of $\mathrm{Gd}^{3+}$ or $\mathrm{La}^{3+}$ for $\mathrm{Eu}^{2+}$ ions in $\mathrm{EuO}$, lead to remarkable increase in $T_{c}$ up to about $170 \mathrm{~K}$ (from $70 \mathrm{~K}$ in stoichiometric $\mathrm{EuO}$ ) and an insulator-to-metal transition (with resistivity change of about 13 orders of magnitude). ${ }^{21,22}$ Similar (but somewhat smaller size) effects also occur in Eu chalcogenides-EuS, EuSe, and EuTe. ${ }^{15}$ The concept of the magnetic polaron is widely employed to explain many remarkable effects in MS and related materials studied by different techniques. ${ }^{23-27}$

So far extensive studies of the formation and dynamics of magnetic polarons have been restricted to a narrow temperature range close to the magnetic phase transition temperature $T_{c}$ (large polaron region). Limited sensitivity (NMR, Raman scattering, etc.) or/and spatial resolution (magneto-optical techniques, neutron scattering, etc.) of many different techniques does not allow detection of magnetic inhomogeneities introduced by small magnetic polarons as MP shrink at temperature well above $T_{c}$. In contrast, the unique sensitivity of polarized positive muons as a local magnetic probe (the muon is sensitive to its immediate environment only) makes muon spin relaxation ( $\mu \mathrm{SR})$ ideally suited for mapping the magnetic state on the atomic (sub-nm) scale. ${ }^{28}$ This approach 
has already contributed greatly to the understanding of magnetic materials, ${ }^{28}$ DMS films, ${ }^{14}$ and bulk DMS materials ${ }^{29}$ as well as bulk MS. ${ }^{30}$ Routine $\mu$ SR studies in condensed matter physics employ highly energetic $4.2 \mathrm{MeV}$ muons with stopping range on the scale of $\mathrm{mm}$ in a solid. The recent development of $\mu \mathrm{SR}$ with a $100 \%$ polarized low energy muon beam (LE- $\mu$ SR) (Ref. 31) of tunable energy between 0.5 and $30 \mathrm{keV}$ (which allows to vary the implantation depth between a few $\mathrm{nm}$ and a few hundred $\mathrm{nm}$ ) provides a powerful tool for mapping magnetic field distributions in thin magnetic films by following the time-dependent $\mu^{+}$spin polarization, that reveals static and dynamical effects of local magnetic fields at the muon site.

In this Brief Report, we present our LE- $\mu$ SR studies of $\mathrm{EuO}$ and Eu chalcogenides films and demonstrate that magnetic polarons do form in these MS around doping center (positive muon) at temperatures well above $T_{c}$ up to room temperature.

Epitaxial films of MS-EuSe, EuTe (both covered by 7 $\mathrm{nm}$ of $\mathrm{Te}$ ), and $\mathrm{EuO}$ (covered by $10 \mathrm{~nm}$ of $\mathrm{Au}$ ) with layer thicknesses of $100 \mathrm{~nm}$ were grown using molecular beam epitaxy on $\mathrm{BaF}_{2}$ (Ref. 32) and $\mathrm{LaAlO}_{3}$, respectively. EuS films of $60 \mathrm{~nm}$ thickness were grown by $e$-beam evaporation on $\mathrm{Si}$ subsrate. X-ray photoemission on the EuO films, collected prior to Au encapsulation, shows $3 d$ and $4 d$ core level line shapes indicative of divalent Eu. X-ray diffraction measurements, after $\mathrm{Au}$ encapsulation, indicate epitaxial and single phase EuO. Superconducting quantum interference device measurements yield magnetization to follow Heisenberg curves with $T_{c}$ of 17 and $70 \mathrm{~K}$ for ferromagnetic EuS and EuO films, respectively; metamagnetic EuSe shows two successive phase transitions at $T_{N}=4.8 \mathrm{~K}$ and $T_{c}=2.7 \mathrm{~K}$ and EuTe enters the AFM state at $T_{N}=9.8 \mathrm{~K}$, all in agreement with literature data. Fully polarized low energy positive muons from the LE muon beam line at the Paul Scherrer Institute (PSI, Switzerland) were implanted into the magnetic layers. Time-differential $\mu \mathrm{SR}$ spectra of the ensemble of about 1-2 million muons were recorded at various temperatures, magnetic fields, and implantation depths.

In the time-differential $\mu \mathrm{SR}$ experiment one accumulates a spectrum of the individual $\mu^{+}$decay events that reveals the time-dependent spin polarization $\mathrm{P}(\mathrm{t})$ of initially $100 \%$ polarized muons stopped in the sample one at a time ${ }^{28}$ in close analogy with free induction decay in NMR. The quantity $\mathrm{P}(\mathrm{t})$ contains the information about the interaction of the muon magnetic moment with its magnetic environment. Each muon stopped in the lattice (typically at an interstitial site) experiences the net effect of external and local magnetic fields and therefore precesses at the characteristic frequency $\omega=\gamma_{\mu} B,\left(\gamma_{\mu}=2 \pi \times 135.5 \mathrm{MHz} / \mathrm{T}\right.$ being the muon gyromagnetic ratio and $B$ is the magnetic field at the muon site). Thus the $\mu$ SR technique offers an extreme sensitivity (often below $0.1 \mathrm{G})$ to the detection of internal magnetic fields of electronic and/or nuclear origin.

In magnetic materials, $\mu^{+} \mathrm{SR}$ measurements have long been employed to determine local magnetic fields at the muon site, as this provides information on the local magnetic structure. ${ }^{28}$ Note that the $\mu$ SR technique requires no application of external magnetic fields as muons arrive $100 \%$ polarized. In the magnetic phase with long-range magnetic order

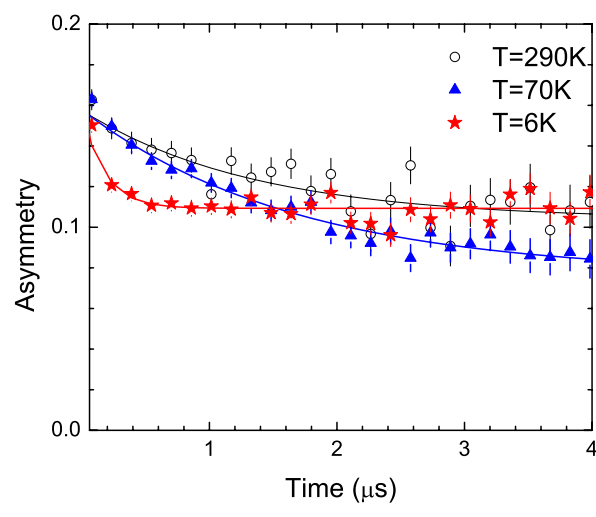

FIG. 1. (Color online) Muon spin relaxation spectra in EuO film at different temperatures below and above $T_{c}=70 \mathrm{~K}$ in zero magnetic field.

(FM or AFM) observation of coherent muon oscillations at a single characteristic frequency $\nu_{\mu}$ in zero applied field $\left(\mathrm{ZF}-\mu^{+} \mathrm{SR}\right)$ gives an unambiguous measure of the local magnetic field at the muon site. If the muons experience a distribution of magnetic fields, $\mathrm{P}(\mathrm{t})$ shows a distribution of precession frequencies (a "lineshape") giving the width of the local magnetic-field distribution $\Delta B$ in a solid. If the distribution is broad when averaged over the sample only a fast relaxation of the muon polarization is observed. By contrast, in a paramagnetic environment the polarization signal exhibits only slow exponential relaxation ("narrow line") because of the fast fluctuations of magnetic moments. In case of different magnetic environments, $\mathrm{P}(\mathrm{t})$ is the superposition of the corresponding signals and it is therefore possible to detect and quantify different magnetic fractions in the material. Details of the technique and data analysis are described in Refs. 28 and 31 .

Figure 1 shows typical ZF- $\mu$ SR spectra in EuO film. Both below and above the FM transition the muon polarization (proportional to the positron decay asymmetry shown on the figure) consists of fast-relaxing and nonrelaxing components.

Such a decomposition of $\mu \mathrm{SR}$ spectra in both $\mathrm{ZF}$ and weak magnetic field transverse to the muon polarization into two components is observed in all the MS films studied at all temperatures from $2 \mathrm{~K}$ up to $300 \mathrm{~K}$. It is found to be independent of the muon stopping depth within the magnetic layer. Temperature dependences of the fast-relaxing components are shown in Figs. 2 and 3.

Observation of the fast-relaxing component at high temperature, deep in the paramagnetic state, where Eu magnetic moments make very fast spin fluctuations (which should lead to at least 1-2 orders of magnitude lower relaxation rates, comparable to nuclear relaxation) is inconsistent with PM environment around the muon (in the PM phase, fast fluctuations of Eu magnetic moments should average out local magnetic field to zero). ${ }^{28}$ Instead, it is consistent with the formation of the MP around the muon, in which the muon finds itself in a FM environment where its relaxation rate is of the electronic origin and should be close to what is found in the experiment. This fast relaxation disappears when the muons are stopped in the nonmagnetic substrate, indicating that it is related with the magnetic environment in Eu-based MS. 


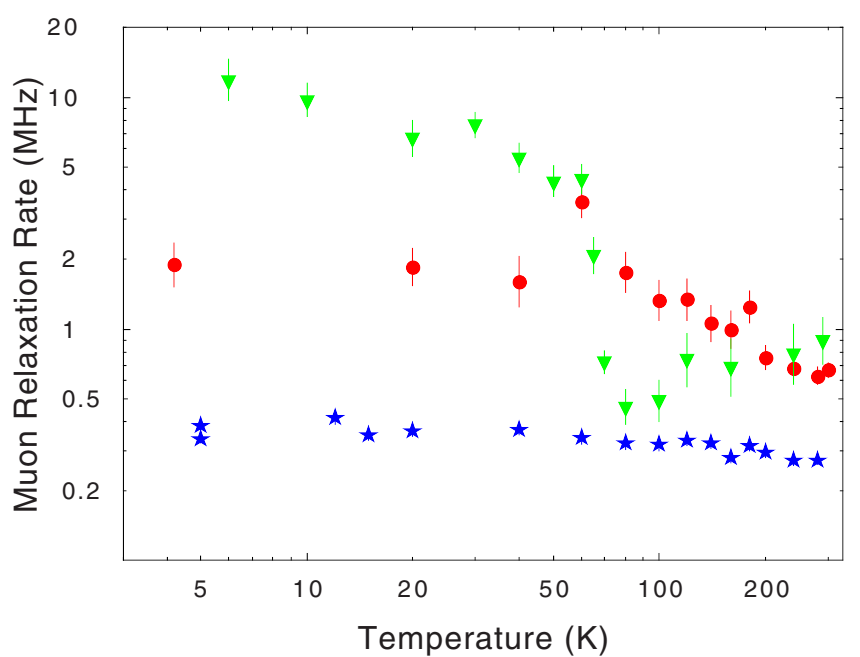

FIG. 2. (Color online) Temperature dependence of the fast muon spin relaxation rate in EuO (triangles, sharp reduction is seen at $T_{c}$ ), EuSe (circles), and EuTe (stars) films in ZF.

Muon spin relaxation due to FM environment in the MP has to be set against another possible relaxation mechanisms: either due to nuclear magnetic moments or due to muonium $\left(\mathrm{Mu} \equiv \mu^{+} e^{-}\right)$atom formation. However, the former is too low to cause the observed relaxation: it is estimated to be no more than $0.1 \mathrm{MHz}$ in all the materials studied. As for $\mathrm{Mu}$, although it presents the source of the electronic relaxation, it has never been observed in the PM environment because of the very rapid spin-exchange mechanism. ${ }^{28}$

Unfortunately, comparatively low magnetic field of the LE muons spectrometer at PSI does not allow spectroscopic detection of the MP. ${ }^{30}$ However, the relaxation rate $\lambda_{f}$ of the fast-relaxing component provides insights into the characteristic local magnetic field which muons feel in the PM phase of Eu-based films: in EuS at room temperature $\lambda_{f}=\gamma_{\mu} \Delta B$ $\approx 2.5 \mathrm{MHz}$ corresponds to $B_{l o c} \sim \Delta B=30 \mathrm{G}$. Such high local magnetic field is consistent with nonfluctuating magnetic

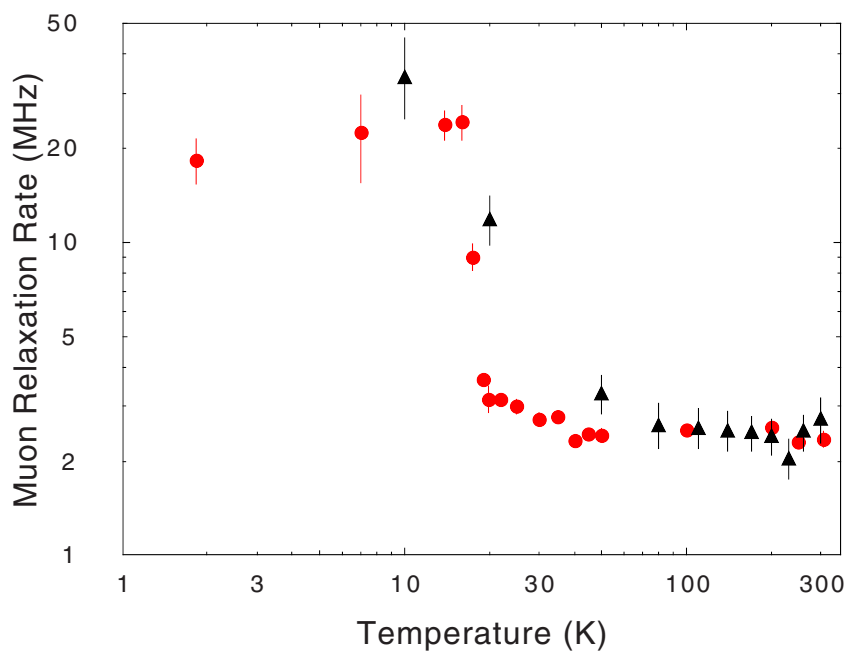

FIG. 3. (Color online) Temperature dependence of the fast muon spin relaxation rate in bulk EuS (circles) and EuS film (triangles). Abrupt change at $T_{c}$ is clearly seen.

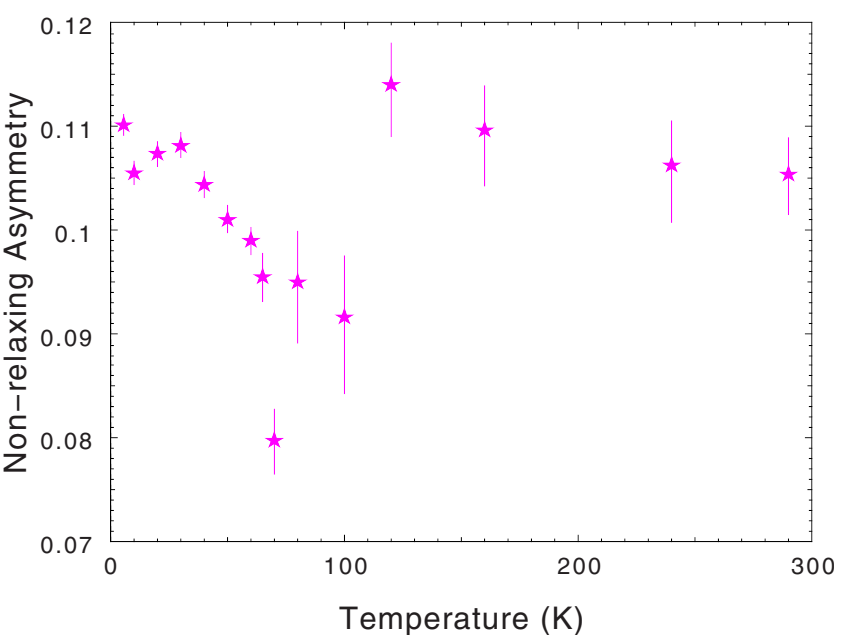

FIG. 4. (Color online) Temperature dependence of the amplitude (asymmetry) of the nonrelaxing component of muon spin polarization in EuO film in zero magnetic field.

environment around the muon. It is in agreement with local magnetic-field shift of about $40 \mathrm{G}$ found in bulk EuS due to MP formation. ${ }^{30}$ Although in EuO, EuSe and EuTe this value is several times less it is still consistent with FM environment around the muon.

Further support for MP formation around the muon comes from the temperature dependence of the amplitude (asymmetry) of the nonrelaxing signal (Fig. 4). It is known that in FM state $1 / 3$ of the muon polarization is concerved in ZF being parallel to local magnetic field of the FM origin (powder approximation). ${ }^{28}$

In MS, MP around the muon is formed when the increase in the electron kinetic energy due to localization is compensated by the combined effect of the exchange coupling and the Coulomb attraction. ${ }^{30}$ The exchange contribution to the localization amounts to a difference between the PM order of the host and the enhanced (FM) order in the MP. Deep in the FM state well below $T_{c}$, the lattice spins are already aligned; the exchange coupling of the carrier with these spins is then of no particular significance and, therefore, electron does not localize into MP and the muon stays "bare." Accordingly, the amplitude of the nonrelaxing component of the muon spin polarization $A_{n}=0.11\left(A_{\text {total }}=0.28\right)$ at low $T$ and follows the Brillouin function as $T$ approaches $T_{c}$ (Fig. 4). The fact that the nonrelaxing component has a minimum around $T_{c}$ indicates that it is related to the magnetic state, but not to any other relaxation mechanisms (such as $\mathrm{Mu}$ formation, etc.). The same value close to $1 / 3$ of the full polarization is found at high $T$ indicating to FM environment around the muon. As the magnetization develops toward $T_{c}$ when temperature is decreased in the PM phase, the exchange contribution to electron localization diminishes and therefore can no longer ensure electron localization into MP which manifests itself as a reduction in the $A_{n}$ around $100 \mathrm{~K}$.

In conclusion, using low-energy muons we found that electrons localize to form magnetic polarons in $\mathrm{EuO}, \mathrm{EuS}$, EuSe, and EuTe films.

This work was partially performed at the Swiss Muon Source of the Paul Scherrer Institute, Switzerland. 
*mussr@triumf.ca

${ }^{1}$ G. A. Prinz, Science 282, 1660 (1998).

${ }^{2}$ S. Datta and B. Das, Appl. Phys. Lett. 56, 665 (1990).

${ }^{3}$ H. C. Koo, J. H. Kwon, J. Eom, J. Chang, S. H. Han, and M. Johnson, Science 325, 1515 (2009).

${ }^{4}$ I. Appelbaum, B. Huang, and D. J. Monsma, Nature (London) 447, 295 (2007).

${ }^{5}$ P. R. Hammar, B. R. Bennett, M. J. Yang, and M. Johnson, Phys. Rev. Lett. 83, 203 (1999).

${ }^{6}$ S. Gardelis, C. G. Smith, C. H. W. Barnes, E. H. Linfield, and D. A. Ritchie, Phys. Rev. B 60, 7764 (1999).

${ }^{7}$ H. J. Zhu, M. Ramsteiner, H. Kostial, M. Wassermeier, H.-P. Schonherr, and K. H. Ploog, Phys. Rev. Lett. 87, 016601 (2001).

${ }^{8}$ G. Schmidt, D. Ferrand, L. W. Molenkamp, A. T. Filip, and B. J. van Wees, Phys. Rev. B 62, R4790 (2000).

${ }^{9}$ R. Fiederling, M. Keim, G. Reuscher, W. Ossau, G. Schmidt, A. Waag, and L. W. Molenkamp, Nature (London) 402, 787 (1999).

${ }^{10}$ Y. Ohno, D. K. Young, B. Beschoten, F. Matsukura, H. Ohno, and D. D. Awschalom, Nature (London) 402, 790 (1999).

${ }^{11}$ H. Ohno, Science 281, 951 (1998).

${ }^{12}$ I. Zutic, Y. Fabian, and S. Das Sarma, Rev. Mod. Phys. 76, 323 (2004).

${ }^{13}$ T. Jungwirth, J. Sinova, J. Masek, J. Kucera, and A. H. MacDonald, Rev. Mod. Phys. 78, 809 (2006).

${ }^{14}$ V. G. Storchak, D. G. Eshchenko, E. Morenzoni, T. Prokscha, A. Suter, X. Liu, and J. K. Furdyna, Phys. Rev. Lett. 101, 027202 (2008).

${ }^{15}$ E. L. Nagaev, Magnetic Semiconductors (Imperial College Press, London, 2002).

${ }^{16}$ J. M. D. Coey, M. Viret, and S. von Molnàr, Adv. Phys. 48, 167 (1999).

${ }^{17}$ P. LeClair, J. K. Ha, H. J. M. Swagten, J. T. Kohlhepp, C. H. van de Vin, and W. J. M. de Jonge, Appl. Phys. Lett. 80, 625 (2002).

${ }^{18}$ T. S. Santos and J. S. Moodera, Phys. Rev. B 69, 241203(R) (2004).

${ }^{19}$ J. Trbovic, C. Ren, P. Xoing, and S. von Molnàr, Appl. Phys. Lett. 87, 082101 (2005).

${ }^{20} \mathrm{~S}$. von Molnár and P. A. Stampe, in Handbook of Magnetism and
Advanced Magnetic Materials, edited by H. Kronmueller and S. Parkin (Wiley, New York, 2007), Vol. 5.

${ }^{21}$ Y. Shapira, S. Foner, and T. Reed, Phys. Rev. B 8, 2299 (1973).

${ }^{22}$ H. Ott, S. J. Heise, R. Sutarto, Z. Hu, C. F. Chang, H. H. Hsieh, H.-J. Lin, C. T. Chen, and L. H. Tjeng, Phys. Rev. B 73, 094407 (2006).

${ }^{23}$ W. Heiss, G. Prechtl, and G. Springholz, Phys. Rev. B 63, 165323 (2001).

${ }^{24}$ H. Rho, C. S. Snow, S. L. Cooper, Z. Fisk, A. Comment, and J.-Ph. Ansermet, Phys. Rev. Lett. 88, 127401 (2002).

${ }^{25}$ A. Comment, J.-Ph. Ansermet, Ch. P. Slichter, H. Rho, C. S. Snow, and S. L. Cooper, Phys. Rev. B 72, 014428 (2005).

${ }^{26} \mathrm{~S}$. Kimura, T. Ito, H. Miyazaki, T. Mizuno, T. Iizuka, and T. Takahashi, Phys. Rev. B 78, 052409 (2008).

${ }^{27}$ J. M. De Teresa, M. R. Ibarra, P. A. Algarabel, C. Ritter, C. Marquina, J. Blasco, J. Garcia, A. del Moral, and Z. Arnold, Nature (London) 386, 256 (1997).

${ }^{28}$ J. H. Brewer, Encyclopedia of Applied Physics Vol. 11 (VCH, New York, 1994), p. 23.

${ }^{29}$ V. G. Storchak, J. H. Brewer, D. G. Eshchenko, S. L. Stubbs, S. P. Cottrell, A. A. Nikonov, and O. E. Parfenov, Spintronics: Materials, Applications and Devices, edited by G. C. Lombardi and G. E. Bianchi (Nova Science Publishers, Inc., New York, 2008), p. 49.

${ }^{30}$ V. G. Storchak, J. H. Brewer, D. J. Arseneau, S. L. Stubbs, O. E. Parfenov, D. G. Eshchenko, E. Morenzoni, and T. G. Aminov, Phys. Rev. B 79, 193205 (2009); V. G. Storchak, O. E. Parfenov, J. H. Brewer, P. L. Russo, S. L. Stubbs, R. L. Lichti, D. G. Eshchenko, E. Morenzoni, T. G. Aminov, V. P. Zlomanov, A. A. Vinokurov, R. L. Kallaher, and S. von Molnár, ibid. 80, 235203 (2009); V. G. Storchak, O. E. Parfenov, J. H. Brewer, P. L. Russo, S. L. Stubbs, R. L. Lichti, D. G. Eshchenko, E. Morenzoni, V. P. Zlomanov, A. A. Vinokurov, and V. G. Bamburov, Phys. B 404, 899 (2009).

${ }^{31}$ E. Morenzoni, F. Kottmann, D. Maden, B. Matthias, M. Meyberg, Th. Prokscha, Th. Wutzke, and U. Zimmermann, Phys. Rev. Lett. 72, 2793 (1994).

${ }^{32}$ R. T. Lechner, G. Springholz, T. U. Schulli, J. Stangl, T. Schwarzl, and G. Bauer, Phys. Rev. Lett. 94, 157201 (2005). 